\title{
Perfil das crianças submetidas à cirurgia cardíaca e abordagem fisioterapêutica em um hospital referência de Salvador
}

\author{
Profile of children undergoing cardiac surgery and physiotherapy approach in a \\ reference hospital of Salvador
}

\author{
Claudiane Ferreira dos Santos ${ }^{1 *}$, Patrícia Abreu Farias Carvalho², Mansueto Gomes Neto ${ }^{3}$, \\ Helena França Correia ${ }^{4 *}$
}

\begin{abstract}
${ }^{1}$ Fisioterapeuta. Mestranda em Processos Interativos de Órgãos e Sistemas pela Universidade Federal da Bahia; ${ }^{2}$ Fisioterapeuta. Especialista em Fisioterapia Cardiovascular; ${ }^{3}$ Doutor em Medicina e Saúde. Docente da pósgraduação em Processos Interativos de Órgão e Sistemas e Medicina e saúde - UFBA; ${ }^{4}$ Doutora em Medicina e Saúde Humana. Docente do Programa de pós-graduação em Processos Interativos de Órgãos e Sistemas - UFBA
\end{abstract}

\begin{abstract}
Resumo
Introdução: o número de crianças que nascem com algum tipo de cardiopatia congênita é elevado e a correção cirúrgica é o principal tratamento nesses casos. A fisioterapia atua com técnicas que auxiliam na recuperação e atenuam disfunções decorrentes do internamento. Objetivo: descrever o perfil clínico de crianças no pós-operatório de cirurgia cardíaca e as principais técnicas fisioterapêuticas utilizadas na Unidade de Terapia Intensiva de um hospital de referência pediátrico do município de Salvador. Metodologia: estudo transversal, com 49 pacientes em hospital de referência em pediatria, submetidos à cirurgia cardíaca no período de novembro de 2016 a agosto de 2017, em hospital de referência em Pediatria. Foram coletados dados dos prontuários referentes a gênero, idade, presença de outras malformações associadas, tipo de cardiopatia, tempo de ventilação mecânica, tempo de internamento na unidade de tratamento intensivo e óbito durante a estada nessa unidade. Resultados: do total da amostra, 53\% eram do sexo feminino. A média de idade foi de 13 meses. Quanto ao tipo de cardiopatia, 85,7\% foram acianóticas, em relação às doenças associadas, $24,4 \%$ eram portadores da Síndrome de Down. Dentre as técnicas fisioterapêuticas mais utilizadas estavam a terapia de higiene brônquica e a cinesioterapia passiva. Conclusão: este estudo demonstrou o perfil clínico dos pacientes no pósoperatório de cirurgias cardíacas, evidenciando uma média de idade de aproximadamente um ano, com discreto predomínio do sexo feminino. As técnicas fisioterapêuticas mais utilizadas foram a terapia de higiene brônquica e a cinesioterapia.

Palavras-chave: Cirurgia torácica. Cardiopatias congênitas. Pediatria.
\end{abstract}

\begin{abstract}
Introduction: the number of children who are born with some kind of congenital heart disease is high and the surgical correction is the main treatment in such cases. Physiotherapy works with techniques that help in the recovery and reduce malfunctions arising from the hospitalization. Objective: describe the clinical profile of children in the postoperative period of cardiac surgery and physiotherapy techniques used in the Intensive Care Unit (ICU) of a reference pediatric hospital of the municipality of. Methodology: cross-sectional study, with 49 patients in pediatric reference hospital who underwent heart surgery in the period from November 2016 to August 2017, in pediatric reference hospital. Data were collected from medical records relating to gender, age, presence of other associated malformations, cardiopathy type, time of VM, time of hospitalization in the ICU and death during a stay in the ICU. Results: of the total sample, 53\% were of female sex. The average age was 13 months. As for the type of cardiopathy, $85.7 \%$ were acyanotic, in relation to associated diseases, $24.4 \%$ were carriers of Down Syndrome. Among the physiotherapeutic techniques used were bronchial hygiene therapy (BHT) and passive kinesitherapy. Conclusion: this study demonstrated the clinical profile of patients in the postoperative period of cardiac surgery, showing an average age of about a year, with discreet predominance of the female sex. The most used physiotherapeutic techniques were the BHT and kinesitherapy.

Keywords: Thoracic Surgery. Congenital Heart Disease. Pediatrics.
\end{abstract}

\section{INTRODUÇÃO}

As cardiopatias congênitas são defeitos anatômicos no coração, presentes ao nascer, que comprometem a funcionalidade do sistema cardiovascular (ARAGÃO et al., 2013). Aproximadamente 8 a cada 1000 nascidos

Correspondente/Corresponding: * Helena França Correia - Instituto de Ciência da Saúde, UFBA. (Departamento de Fisioterapia) - End: Av. Reitor Miguel Calmon, s/n, Vale do Canela, $4^{\circ}$ andar, sala 422 CEP 40.110-902 Salvador, Ba - Tel: (71) 3283-8910 - E-mail: lenafran@gmail.com vivos apresentam algum tipo de anomalia cardíaca ao nascer e apenas $20 \%$ desses defeitos são resolvidos sem nenhum tipo de intervenção, pois o restante precisará de correção cirúrgica. Estima-se que, por ano, sejam realizados 23.077 procedimentos cirúrgicos no Brasil (PINTO JÚNIOR et al., 2015).

Usualmente, as cardiopatias congênitas podem ser classificadas em acianóticas e cianóticas. As cardiopatias acianóticas são mais frequentes e sua correção requer um nível de complexidade menor em relação às car- 
diopatias cianóticas. Entre as cardiopatias acianóticas mais frequentes estão: a comunicação interatrial (CIA), a comunicação interventricular (CIV), defeito no septo atrioventricular total (DSAVT) ou parcial (DSAVP), que muitas vezes pode estar associado com a síndrome de Down, a estenose aórtica, persistência do canal arterial (PCA) e a coarctação da aorta (CoA). As cardiopatias congênitas cianóticas implicam uma maior gravidade e a mais comum é a tetralogia de Fallot (T4F), correspondendo a 10\% de todas as cardiopatias (BUENO; KIMURA, 2008).

A correção cirúrgica pode ser paliativa ou corretiva, com base na anatomia cardíaca e no quadro clínico da criança, porém, recomenda-se que a cirurgia corretiva seja realizada o mais breve possível para eliminar possíveis efeitos das lesões não operadas, como a insuficiência cardíaca congestiva e infecções respiratórias de repetição (PINTO JÚNIOR et al., 2015).

O pós-operatório de pacientes submetidos à cirurgia cardíaca requer acompanhamento em Unidade de Terapia Intensiva (UTI), sendo que a maioria necessita de ventilação mecânica (VM). A assistência da equipe multidisciplinar é imprescindível para atenuar e tratar complicações durante o reestabelecimento dos pacientes. (SILVA et al., 2008).

A fisioterapia dentro da UTI, em conjunto com a equipe multidisciplinar, tem como objetivo intervir nas complicações pulmonares (retenção de secreções pulmonares, atelectasias e pneumonias), contribuindo para uma ventilação adequada, para a extubação bem sucedida e um posicionamento no leito favorável, com o intuito de reduzir os riscos associados ao tempo de internamento e do uso prolongado de ventilação mecânica; ademais, promove a mobilização precoce que contribui para a redução dos déficits motores causados pela imobilidade no leito. (SOARES; SILVA; CALLES, 2017).

Conhecer o perfil da população submetida a cirurgias cardíacas pediátricas contribui para elaboração de um plano assistencial, promovendo condições favoráveis para a recuperação desses pacientes. Sendo assim, este estudo teve como objetivo descrever o perfil clínico de crianças no pós-operatório de cirurgia cardíaca e as principais técnicas fisioterapêuticas utilizadas na UTI de um hospital de referência pediátrico do município de Salvador.

\section{METODOLOGIA}

Trata-se de um estudo transversal, conduzido entre novembro de 2016 e agosto de 2017 com pacientes no pós-operatório de cirurgia cardíaca, admitidos na UTI do Hospital Martagão Gesteira, centro de referência em Pediatria, situado na cidade de Salvador (BA). Esta pesquisa foi aprovada pelo Comitê de Ética e Pesquisa, do Instituto de Ciências da Saúde, da Universidade Federal da Bahia, pelo Parecer no 1.813.728, com dispensa do Termo de Consentimento Livre e Esclarecido.
Incluíram-se pacientes com idade entre 29 dias a 6 anos e 11 meses, de ambos os sexos, que realizaram correção cirúrgica de cardiopatias congênitas e tiveram o pós-operatório acompanhado em uma das duas Unidades de Terapia Intensiva Pediátrica até a sua alta da UTI. Foram excluídos pacientes cujo dados dos prontuários estavam incompletos ou que foram transferidos para outra unidade.

Foram coletados dados dos pacientes internados que preencheram os critérios de inclusão, dentro do período especificado, até sua alta ou óbito da UTI. As seguintes variáveis foram coletadas e analisadas: idade, sexo, diagnóstico(s) pré-operatório(s), tipo de intervenção e incisão cirúrgica, assim como complicações pós-operatórias.

As condutas fisioterapêuticas foram divididas entre pré-extubação e pós-extubação. Os procedimentos registrados na pré-extubação incluíram: ajustes ventilatórios para manter uma oxigenação satisfatória e $\mathrm{SpO}_{2}$ acima de $90 \%$; terapia de higiene brônquica (THB), com aspiração endotraqueal; hiperinsuflação manual; posicionamento no leito, mudança de decúbito e exercícios passivos para manutenção da amplitude articular. As condutas pós-extubação foram: manobras manuais desobstrutivas e reexpansivas, THB, ventilação não invasiva (VNI), utilizada quando o paciente apresentava insuficiência respiratória, atelectasia, hipoventilação, entre outros. $E$, ainda, inspirometria de incentivo (EI), dispositivo de pressão positiva expiratória (EPAP) e cinesioterapia, incluindo deambulação precoce (FELCAR et al., 2008).

A estatística descritiva foi utilizada para apresentação dos resultados. As variáveis categóricas foram expressas em frequências absolutas e relativas. Para as variáveis numéricas foram utilizados uma medida de tendência central (média e mediana) e sua variabilidade (desvio padrão e intervalo interquartil), conforme distribuição dos dados. Os dados foram analisados no Statistical Package for the Social Sciences, versão 20.0 (SPSS Inc., Chicago, IL, EUA).

\section{RESULTADOS}

Foram colhidos dados de 49 pacientes submetidos à cirurgia cardíaca para correção de cardiopatias congênitas. A idade variou de 2 meses a 6 anos e 10 meses, sendo que a mediana, em meses, foi de $13(9,25-29,25)$, havendo um predomínio do sexo feminino (53\%). Quanto ao tipo de cardiopatia, $85,7 \%$ foram acianogênicas. 0 diagnóstico mais frequente foi a associação de algumas cardiopatias (42,9,3\%). Isoladamente, a CIV e a PCA foram as mais frequentes, com $14,3 \%$ cada uma. Vinte e quatro por cento eram portadores da Síndrome de Down. As características demográficas e clínicas da população são apresentadas na Tabela 1. 
Tabela 1 - Características demográficas e clínicas da população

\begin{tabular}{lr}
\hline VARIÁVEIS & $\mathbf{n}=49$ \\
\hline Idade (meses), mediana (IQ) & $26(53)$ \\
Sexo feminino, $\mathrm{n}(\%)$ & \\
Tipo de cardiopatia, $\mathrm{n}(\%)$ & $7(14,3)$ \\
$\quad$ Cianótica & $42(85,7)$ \\
Acianótica & $34(69,4)$ \\
Esternotomia mediana, $\mathrm{n}(\%)$ & \\
Doenças associadas, $\mathrm{n}(\%)$ & $12(24,4)$ \\
Síndrome de Down & $6(12,2)$ \\
Síndromes genéticas diversas & $4(8,1)$ \\
Outros & \\
Diagnóstico, $\mathrm{n}$ (\%) & $21(42,9)$ \\
Cardiopatias associadas & $7(14,3)$ \\
CIV & $7(14,3)$ \\
PCA & $6(12,2)$ \\
TF4 & $1(2,0)$ \\
Insuficiência valvar & $3(6,1)$ \\
DSAV & $2(4,1)$ \\
Coarctação da aorta & $2(4,1)$ \\
CIA & $34(69,3)$ \\
Esternotomia mediana, $\mathrm{n}$ (\%) & \\
Tempo de CEC em minutos, mediana (IQ) & $(29,7-81,2)$ \\
Local da extubação, $\mathrm{n}$ (\%) & \\
CC & $6(12,2)$ \\
UTI & $43(87,7)$ \\
\hline
\end{tabular}

Fonte: Dados da pesquisa

Legenda: CIA - comunicação interatrial; CIV - comunicação interventricular; PCA - persistência do canal arterial; DSAV - defeito do septo atrioventricular, tetralogia de Fallot; CEC - circulação extracorpórea; CC - centro cirúrgico; UTI - unidade de terapia intensiva, VM - ventilação mecânica.

Na Tabela 2, são ilustrados os desfechos clínicos na UTI e a ocorrência de complicações no pós-operatório de cirurgia cardíaca pediátrica. A principal complicação no pós-operatório foram as pulmonares, com uma incidência de $69,4 \%$, seguidas das complicações neurológias e sepse, com $8,1 \%$ cada.

Tabela 2 - Desfechos clínicos na UTI e complicações pulmonares no pós-operatório de cirurgia cardíaca pediátrica

\begin{tabular}{lr}
\hline VARIÁVEIS & $\mathbf{N ~ ( \% )}$ \\
\hline Falha de extubação, n (\%) & $6(12,2)$ \\
Uso de VNI após extubação, n (\%) & $11(22,4)$ \\
Tempo de UTI em dias, mediana (IQ) & $7,5(5,2-30,0)$ \\
Complicações pós-operatórias n (\%) & \\
Complicações pulmonares & $34(69,4)$ \\
Complicações neurológicas & $4(8,1)$ \\
Complicações cardíacas & $3(6,1)$ \\
Insuficiência renal & $3(6,1)$ \\
Sepse & $4(8,1)$ \\
SRIS & $3(6,1)$ \\
Tempo de VM $\geq 24 h, n(\%)$ & $25(51,0)$ \\
Tempo de UTI em dias, mediana (IQ) & $2(4,1)$ \\
Óbito, n (\%) & $7,2-30,0)$ \\
\hline
\end{tabular}

Fonte: Dados da pesquisa
Legenda: VM - ventilação mecânica; VNI - ventilação não invasiva, SDRA - síndrome do desconforto respiratório agudo; SRIS - síndrome da resposta inflamatória sistêmica;IQ - intervalo interquartil; UTI unidade de terapia intensiva

Apenas 6 pacientes não receberam atendimento fisioterapêutico pré-extubação por terem sido extubados no centro cirúrgico, sendo as condutas mais frequentes a cinesioterapia e a terapia de higiene brônquica em 43 pacientes $(100 \%)$, seguidas das manobras manuais desobstrutivas em 15 deles (30,6\%), hiperinsuflação manual em 10 (20,4\%), terapia de expansão pulmonar (manobra de recrutamento alveolar - MRA) em 8 (16,3\%) e apenas $6(12,2 \%)$ realizaram manobras manuais reexpansivas. Estes dados estão evidentes na Figura 1

Figura 1 - Condutas fisioterapêuticas realizadas pré-extubação no pós-operatório de cirurgia cardíaca pediátrica.

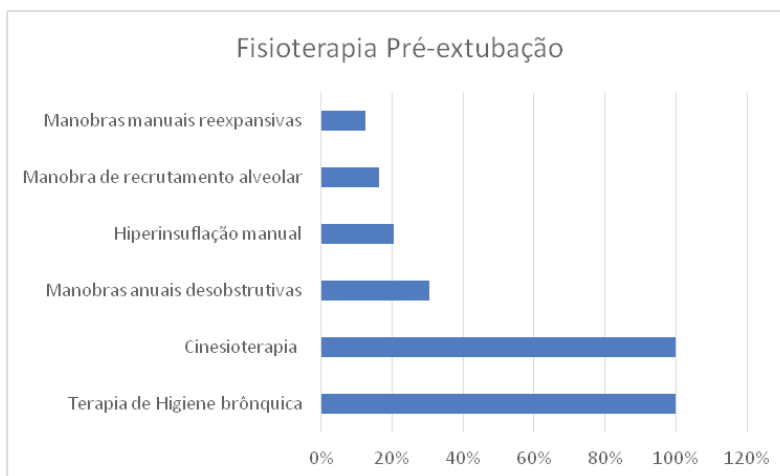

Fonte: Dados da pesquisa.

Com relação às condutas fisioterapêuticas pós-extubação, todos os pacientes da amostra foram submetidos à cinesioterapia; já à terapia de higiene brônquica, 47 $(95,9 \%)$, exceto os 2 pacientes que foram a óbito; 24 $(48,9 \%)$ receberam manobras manuais desobstrutivas, $11(22,4 \%)$ ventilação não invasiva, $12(24,4 \%)$ manobras reexpansivas e apenas $1(2,04 \%)$ foi submetido a um dispositivo de pressão positiva expiratória (EPAP). Estes dados encontram-se presentes na Figura 2.

Figura 2 - Condutas fisioterapêuticas realizadas pós-extubação no pós-operatório de cirurgia cardíaca pediátrica.

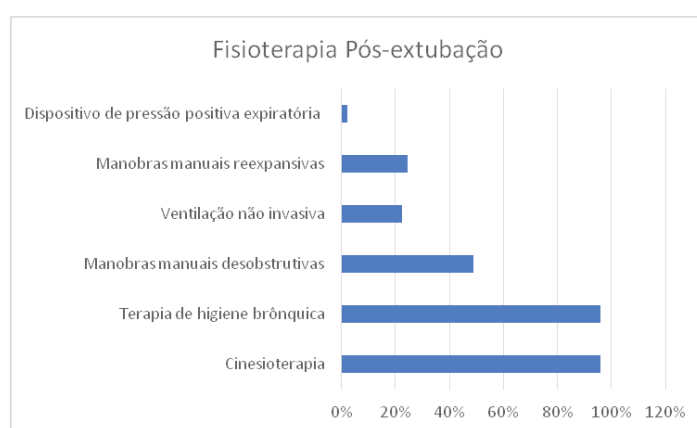

Fonte: Dados da pesquisa. 


\section{DISCUSSÃO}

O número de cirurgias realizadas para correção de cardiopatias congênitas vem aumentando, isto se deve principalmente às repercussões hemodinâmicas causadas pelo hiperfluxo ou hipofluxo pulmonar, dependendo do tipo de cardiopatia. Das 49 cirurgias realizadas no período do estudo, houve um discreto predomínio do sexo feminino de $53 \%$, achado semelhante ao estudo de Barros, Dias e Nina (2014) que encontraram uma predominância do sexo feminino, com percentual de $53,68 \%$. Outros autores também encontraram uma frequência maior no sexo feminino (BELO; OSELAME; NEVES, 2016).

Em relação à faixa etária, a idade média encontrada nesta análise foi de 13 meses, compatível com o estudo de Aragão et al. (2013), em que a faixa etária se manteve entre 31 dias e 2 anos de idade. $O$ estudo Shah et al. (2016) encontrou uma frequência de cirurgias cardíacas maior em pacientes com idade média de 22,31 $\pm 34,08$ meses.

A prevalência de cardiopatias acianóticas é evidente em vários estudos. Aragão et al. (2013) verificaram em seu estudo que $70 \%$ das cardiopatias congênitas foram acianóticas, sendo a mais frequente a comunicação intraventricular (21\%). Shah et al.(2016) observaram que a cardiopatia congênita acianótica foi a mais frequente $(81,5 \%)$, seguida por cardiopatia congênita cianótica $(14,2 \%)$; esses achados na literatura são compatíveis com os dados analisados neste estudo em que as cardiopatias acianóticas corresponderam a aproximadamente $86 \%$ da amostra.

Dentre as cardiopatias cianóticas, a anomalia mais frequente foi a tetralogia de Fallot (12,2\%). No estudo de Carmo et al. (2017), 5,7\% da amostra apresentavam este tipo de cardiopatia. Outros autores encontraram uma frequência maior para transposição de grandes artérias (34,3\%) e $25,8 \%$ para tetralogia de Fallot (GUITTI, 2000; MIYAGUE et al., 2003). Porém, a instituição onde foi realizada a coleta admite apenas cirurgias de médio a baixo nível de complexidade, o que justifica a falta de ocorrência de outras cardiopatias cianóticas, em que a cirurgia exige um nível de complexidade mais elevado.

A associação das cardiopatias congênitas com outras anomalias tem sido debatida na literatura; diversas síndromes genéticas podem estar presentes em pacientes com cardiopatias congênitas, sendo que Aragão et al. (2013) encontraram uma associação de $4 \%$ com a Síndrome de Down. Neste estudo, $24,4 \%$ das crianças eram portadoras dessa síndrome e $12,2 \%$ eram portadoras de outras síndromes genéticas.

O tempo de internação variou de 5 até 30 dias, com uma média de 7 dias, o que difere do estudo de Belo, Oselame e Neves (2016), que obteve uma média de 119 dias. Fatores como idade, peso, tipo de cardiopatia e complicações sistêmicas no pós-operatório impactam diretamente na ocorrência de óbito no pós-operatório de cirurgias cardíacas pediátricas. Barros, Dias e Nina (2014) identificaram, em seu estudo, uma mortalidade de $11,1 \%$, atribuindo-a à complexidade da cardiopatia. Nesta amostra, ocorreram dois óbitos associados a complicações sistêmicas, sendo que um era portador de cardiopatia cianótica. No estudo de Aragão et al. (2013), 83\% dos pacientes com cardiopatias cianóticas evoluíram para óbito. A taxa de mortalidade de pacientes com esse tipo de cardiopatia, no estudo de Nina et al. (2007), foi menor, correspondendo a $66 \%$ de óbitos, sendo uma das causas a infecção pulmonar.

A fisioterapia, no pós-operatório de cirurgia cardíaca tem sido utilizada com o objetivo de prevenir e tratar complicações pulmonares, como atelectasias e acúmulo de secreções pulmonares. As técnicas descritas neste estudo foram divididas em pré e pós-extubação, sendo que os pacientes extubados no centro cirúrgico não realizaram fisioterapia pré-extubação. Todos os pacientes em VM receberam ajustes ventilatórios, visando a assegurar uma função pulmonar adequada e a extubação com maior brevidade possível. O recomendado é que as crianças em VM, no pós-operatório de cirurgias cardíacas, sejam extubadas assim que cesse o efeito da narcose anestésica (FELCAR et al., 2008). Neste estudo, a maioria das crianças foi extubada até 24 horas após a intubação.

As técnicas fisioterapêuticas mais empregadas pré-extubação, neste estudo, foram a THB, cujo objetivo é evitar o acúmulo de secreções traqueobrônquicas, e a cinesioterapia passiva que inclui o posicionamento correto no leito, com o objetivo de reduzir a rigidez, dores musculares e preservar a amplitude articular. Essas técnicas são consideradas importantes para melhora da relação ventilação perfusão no pós-operatório de cirurgia torácica (SASSERON et al., 2009). As manobras manuais desobstrutivas e reexpansivas são utilizadas em menor frequência, possivelmente por conta da ferida operatória, pois estas consistem em compressões torácicas (MIRANDA; PADULLA; BORTOLATTO, 2011). A terapia de expansão pulmonar com a manobra de recrutamento alveolar foi realizada em $17,2 \%$ dos pacientes entubados. Esta manobra tem a função de recrutar alvéolos colapsados, melhorando a relação ventilação perfusão (PADOVANI; CAVENAGHI, 2011).

Após a extubação, o objetivo da fisioterapia é a manutenção da ventilação espontânea do paciente (MIRANDA; PADULLA; BORTOLATTO, 2011). Estão entre as técnicas fisioterapêuticas empregadas com maior frequência: THB, VNI, inspirômetro de incentivo, EPAP e cinesioterapia ativa, que inclui sedestação e deambulação precoce (FELCAR et al., 2008). Todos os pacientes deste estudo realizaram fisioterapia motora e as manobras manuais desobstrutivas foram utilizadas por metade dos pacientes, com o objetivo de evitar o acúmulo de secreções, manter a função pulmonar e evitar complicações pulmonares. As manobras manuais reexpansivas foram utilizadas em uma frequência menor. A VNI foi empregada nos casos de atelectasia. A fisioterapia pode prevenir a deterioração da função pulmonar e reduzir a incidência de complicações pulmonares. Dessa forma, as técnicas vêm sendo empregadas em pacientes no pós-operatório de cirurgia cardíaca (SILVA et al., 2011). 


\section{Limitações do estudo}

Este estudo foi realizado em um único centro de referência, porém os resultados apresentados são condizentes com a literatura encontrada. A coleta foi realizada mediante a revisão em prontuário eletrônico, deixando o pesquisador dependente dos dados registrados pelos profissionais e dificultando a identificação de situações clínicas omitidas ou subnotificadas, embora todos os cuidados fossem tomados para garantir a melhor qualidade da informação.

\section{CONCLUSÃO.}

Este estudo apresentou o perfil clínico dos pacientes no pós-operatório de cirurgias cardíacas, evidenciando uma média de idade de aproximadamente um ano, com discreto predomínio do sexo feminino. As cardiopatias acianóticas foram as mais frequentes e os achados apontaram uma associação com algumas síndromes genéticas, sendo a principal delas a síndrome de Down. As técnicas fisioterapêuticas mais utilizadas foram a terapia de higiene brônquica e a cinesioterapia.

\section{Potencial conflito de interesses}

Declaro não haver conflitos de interesses.

\section{Fontes de financiamento}

Esse estudo foi financiado com recursos próprios do investigador.

\section{REFERÊNCIAS}

ARAGÃO, J. A. et al. O Perfil epidemiológico dos pacientes com cardiopatias congênitas submetidos à cirurgia no hospital do coração. Revista Brasileira de Ciências da Saúde, Aracaju, v. 17, n. 3, p.263-268, 30 out. 2013. Disponível em: <http://www.periodicos.ufpb.br/index.php/rbcs/article/ view/13221>. Acesso em: 19 ago. 2018. DOI: 10.4034/rbcs.2013.17.03.08

BELO, W. A.; OSELAME, G. B.; NEVES, E. B. Perfil clínico-hospitalar de crianças com cardiopatia congênita. Cadernos de Saúde Coletiva, Rio de Janeiro, v. 10, n 12, p. 312-319, 2016. Disponível em: <http://www.scielo. com.br>. Acesso em: 20 de ago. 2018

BARROS, T. L. do V.; DIAS, M. de J. S.; NINA, R. V. de A. H. Congenital cardiac disease in childhood $x$ socioeconomic conditions: a relationship to be considered in public health? Revista Brasileira de Cirurgia Cardiovascular, São Paulo, v. 29, n. 3, p. 448-454, 2014. Disponível em: <http:// www.scielo.com.br>. Acesso em: 20 de ago. 2018

BUENO, M.; KIMURA, A. F. Perfil de recém-nascidos submetidos à cirurgia cardíaca em hospital privado do Município de São Paulo. Revista de Enfermagem USP, São Paulo, v. 42, n.1, p.112-119. 2008. Disponível em: <http://www.scielo.com.br> Acesso em; 20 ago. 2018.

CARMO, L. A. do et al. Perfil clínico e epidemiológico das crianças com cardiopatias congênitas atendidas pela rede de cardiologia pediátrica pernambuco - paraíba. Revista Interdisciplinar em Saúde, Pernanbuco, v. 5 n.1, jan./mar. 2017. Disponível em: <http://www.scielo.com.br> Acesso: em 20 ago. 2018
FELCAR, J. M. et al. Fisioterapia pré-operatória na prevenção das complicações pulmonares em cirurgia cardíaca pediátrica. Revista Brasileira de Cirurgia Cardiovascular, Curitiba, v. 23, n. 3, p.383-388, set. 2008. Disponível em: $<$ http://www.scielo.br/scielo.php?script=sci_arttext\&pid $=$ S0102-76382008000300016>. Acesso em: 01 ago. 2018. DOI: 10.1590/ s0102-76382008000300016.

GUITTI, J. C. S. Epidemiological characteristics of congenital heart diseases in Londrina. Arquivos Brasileiros de Cardiologia, Curitiba, v.74, n.5, p 400-404, 2000. Disponível em: <https://www.researchgate.net/profile/>. Acesso em: 01 ago. 2018.

MIRANDA, R. C. V. de; PADULLA, S. A. T.; BORTOLATTO, C. R. Fisioterapia respiratória e sua aplicabilidade no período pré-operatório de cirurgia cardíaca. Revista Brasileira de Cirurgia Cardiovascular, São Paulo, v. 26, n. 4, p.647-652, 2011. Disponível em: <http://www.scielo.br/scielo>. Acesso em: 04 ago. 2018. DOI: 10.5935/1678-9741.20110057.

MIYAGUE, N. I. et al. Estudo epidemiológico de cardiopatias congênitas na infância e adolescência: Análise em 4.538 casos. Arquivos Brasileiros de Cardiologia, Curitiba, v. 80, n. 3, p 269-273, 2003. Disponível em: <http:// www.scielo.br/pdf/abc>. Acesso em: 04 ago. 2018.

NINA, R. V. A. H. et al. O escore de risco ajustado para cirurgia em cardiopatias congênitas (RACHS-1) pode ser aplicado em nosso meio? Revista Brasileira de Cirurgia Cardiovascular. São Paulo, v. 22, n.4, 2007. Disponível em: <http://www.scielo.br/scielo>. Acesso em: 4 ago. 2018.

PADOVANI, C.; CAVENAGHI, O. M. Recrutamento alveolar em pacientes no pós-operatório imediato de cirurgia cardíaca. Revista Brasileira de Cirurgia Cardiovascular, São Paulo, v. 26, n. 1, p.116-121, nov. 2011. Disponível em: <http://www.scielo.br/pdf/>. Acesso em: 19 ago. 2018.

PINTO JÚNIOR, V. C. et al. Epidemiology of congenital heart disease in Brazil Approximation of the official Brazilian data with the literature. Revista Brasileira de Cirurgia Cardiovascular, São Paulo, p.219-224, fev. 2015. Disponível em: <http://www.scielo.br/scielo.php?. Acesso em: 21 ago. 2018. DOI: 10.5935/1678-9741.20150018.

SASSERON, A. B. et al. A dor interfere na função respiratória após cirurgias cardíacas? Revista Brasileira de Cirurgia Cardiovascular, São Paulo, v. 24, n. 4, p.490-496, dez. 2009. Disponível em: <http://www.scielo.br/scielo>. Acesso em: 4 ago. 2018. DOI: 10.1590/s0102-76382009000500010.

SHAH, P. et al. Spectrum of Congenital Heart Diseases in Eastern Nepal: A tertiary care hospital experience. Journal of College of Medical Sciences, Nepal, v.12, n. 4, Oct-Dec 2016. Disponível em: <http://www.scielo.br/ scielo.php?. Acesso em: 21 ago. 2018.

SILVA, M. E. M. da et al. Cirurgia cardíaca pediátrica: o que esperar da intervenção fisioterapêutica?. Revista Brasileira de Cirurgia Cardiovascular, São Paulo, v. 26, n. 2, p.264-272, jun. 2011. Disponível em: <http://www.scielo.br/pdf/>. Acesso em: 18 ago. 2018. DOI: 10.1590/ s0102-76382011000200018.

SILVA, Z. M. et al. Fatores associados ao insucesso no desmame ventilatório de crianças submetidas a cirurgia cardíaca pediátrica. Revista Brasileira de Cirurgia Cardiovascular, Porto Alegre, v. 23, n. 4, p.501-506, set. 2008. Disponível em: <http://www.scielo.br/pdf/rbccv/v23n4/v23n4a08>. Acesso em: 20 ago. 2018

SOARES, E. de M.; SILVA, C. C. M. da; CALLES, A. C. do N. Incidência das complicações pulmonares em cirurgias cardiopediátricas. Ciências Biológicas e de Saúde Unit, Maceió, v. 4, n. 2, p.313-322, nov. 2017. Disponível em: <https://periodicos.set.edu.br/index.php/fitsbiosaude>. Acesso em: 17 ago. 2018

Submetido em: $14 / 11 / 218$

Aceito em: 29/11/2018 